\title{
The Principal Principle, admissibility, and normal informal standards of what is reasonable
}

\author{
Jürgen Landes ${ }^{1}$ (D) . Christian Wallmann ${ }^{2}$. Jon Williamson ${ }^{3}$ iD
}

Received: 16 June 2020 / Accepted: 29 January 2021 / Published online: 27 March 2021

(C) The Author(s) 2021

\section{Abstract}

This paper highlights the role of Lewis' Principal Principle and certain auxiliary conditions on admissibility as serving to explicate normal informal standards of what is reasonable. These considerations motivate the presuppositions of the argument that the Principal Principle implies the Principle of Indifference, put forward by Hawthorne et al. (British Journal for the Philosophy of Science, 68, 123-131, 2017). They also suggest a line of response to recent criticisms of that argument, due to Pettigrew (British Journal for the Philosophy of Science, 71, 605-619, 2020) and Titelbaum and Hart (British Journal for the Philosophy of Science, 71(2), 621-632, 2020). The paper also shows that related concerns of Hart and Titelbaum (Thought: A Journal of Philosophy, 4(4), 252-262, 2015) do not undermine the argument of Hawthorne et al. (2017).

Keywords Principal Principle $\cdot$ Bayesianism $\cdot$ Admissibility $\cdot$ Chance $\cdot$ Indifference

\section{Introduction}

Our personal beliefs should be responsive to our evidence. For example, if a coin will be tossed and the coin is a fair coin, according to our evidence, then we ought to be

Jürgen Landes

juergen_landes@yahoo.de

Christian Wallmann

Christian.Wallmann@fh-steyr.at

Jon Williamson

j.williamson@kent.ac.uk

1 Munich Center for Mathematical Philosophy, Faculty of Philosophy, Philosophy of Science and Study of Religion, LMU Munich, München, Germany

2 Josef Ressel Centre for Real-time Value Network Visibility, Logistikum, University of Applied Sciences Upper Austria, Wels, Austria

3 Centre for Reasoning and Department of Philosophy, University of Kent, Canterbury, UK 
equally confident that it will land heads as tails. Bayesian epistemology captures such claims by means of principles of rationality that link degrees of belief to objective probabilities (often called chances). The most prominent of these principles, David Lewis' Principal Principle, says that one ought to believe a proposition $A$, conditional on the chance of $A$ being $x$ and other admissible information, to degree $x$.

There has been a lot of interesting work on the Principal Principle in recent years. Some researchers have identified problems with the principle: for example, it has been argued that the Principal Principle conflicts with the requirement to have accurate degrees of belief (Easwaran and Fitelson 2012) and that it creates tensions with the claim that conditional beliefs are conditional probabilities (Wallmann and Hawthorne 2018; Wallmann and Williamson 2020). Further topics of interest have included the notion of admissibility (Meacham 2010) and the relation between the Principal Principle and Humeanism (Black 1998). A number of other chance-credence calibration principles have been proposed (Hall 1994; Vranas 2004).

In this paper, we pick up on the argument of Hawthorne et al. (2017), which shows that, given certain auxiliary conditions about the admissibility of additional information, the Principal Principle implies a version of the Principle of Indifference: it implies that a contingent atomic proposition should be believed to degree $\frac{1}{2}$, in the absence of evidence that bears on that proposition. This result is surprising because it suggests that the Principal Principle requires an objective Bayesian framework (in which degrees of belief are highly constrained in the absence of relevant evidence) rather than the usual subjective Bayesian framework, and because the Principle of Indifference is much more controversial than the Principal Principle.

One often-voiced criticism of the Principle of Indifference is that it results in contradictions when the underlying state space is changed (Seidenfeld 1987; Norton 2019). Present-day proponents simply accept that uncertain inference depends on the choice of an appropriate state space (Paris 2014; Halpern and Koller 2004; Novack 2010; Williamson 2010) and point out that the principle is consistent under uniform refinements of the underlying state space (Paris 2006). Recent work has focussed on tensions between the Principle of Indifference and imprecise probabilities (Rinard 2014; White 2010) and self-locating beliefs (Marcoci 2020). New justifications of the Principle of Indifference continue to emerge, e.g., utilising Carnap's conceptual spaces (Decock et al. 2016), accuracy first epistemology (Pettigrew 2016) and consequentialism (Williamson 2018). It has even been argued that the Principle of Indifference supports inductive scepticism (Smithson 2017).

The argument of Hawthorne et al. (2017) has been roundly criticised by Pettigrew (2020) and Titelbaum and Hart (2020), who suggest that the conditions on admissibility that it invokes are unwarranted. Here, we defend the argument of Hawthorne et al., by showing that its auxiliary conditions on admissibility are warranted by precisely the same considerations that warrant David Lewis' Principal Principle itself: normal informal standards of what is reasonable.

The paper is structured as follows. In Section 2 we show how normal informal standards of what is reasonable motivate the Principal Principle and its auxiliary conditions on admissibility. The argument of Hawthorne et al. (2017) that the Principal Principle implies the Principle of Indifference is defended against criticisms of 
Pettigrew in Section 3 and those of Titelbaum and Hart in Section 4. In Section 5 we briefly consider two further objections and conclude that the Principal Principle does indeed imply the Principle of Indifference.

\section{Normal informal standards of what is reasonable}

\subsection{Questionnaire}

Imagine you are a goat farmer, interested in the colour of the next goat to be born to your herd. Your evidence determines that the chance of that goat-Ashley, saybeing brown $(A)$ is 0.7 . Consider four alternative scenarios, and ask yourself in each case what degree of belief in $A$ would be reasonable:

(a) You have no further evidence.

Answer. Degree 0.7 stands out as uniquely reasonable.

(b) You do have further evidence, namely some contingent atomic proposition $F$ (e.g., the proposition that Finley, another goat, escapes).

Answer. Still 0.7. Without any evidence that relates $F$ to $A$, you have no grounds for any other choice. The chance of $A$ gives robust grounds for believing $A$ to degree 0.7 .

(c) You have instead evidence just that $A \leftrightarrow F$, for some contingent atomic proposition $F$ (e.g., $F$ says that Francis is brown and you learn that Ashley and Francis are identical twins, so $A \leftrightarrow F)$.

Answer. Still 0.7. With no other evidence bearing on $F$, learning that $A$ and $F$ have the same truth value doesn't tell you anything about $A$.

(d) You have instead evidence just that $A \leftrightarrow F$, for some complex $F$ (e.g., $F$ says that Fiona and Felix and Freya and Fernando are all brown, $F=F_{1} \wedge F_{2} \wedge F_{3} \wedge$ $F_{4}$, and you learn $A \leftrightarrow F$ ).

Answer. This isn't obvious. There's no external evidence bearing on $F$, but one might think that a conjunction of atomic propositions is inherently somewhat less credible. So, one might think that there is information here that modulates the influence of the chance on one's degree of belief. A degree of belief lower than 0.7 might be reasonable.

\subsection{The Principal Principle}

On the basis of a similar questionnaire, David Lewis (1986) put forward the following principle as a constraint on a reasonable initial credence function $P$, which is taken to be a probability function:

Principal Principle. $P(A \mid X E)=x$, where $X$ says that the chance at time $t$ of proposition $A$ is $x$ and $E$ is any proposition that is compatible with $X$ and admissible at time $t$.

Lewis' Principal Principle requires auxiliary conditions that specify certain propositions as admissible. These auxiliary conditions are crucial, because the Principal 
Principle can only apply in cases where potential evidence $E$ is admissible. As Lewis notes,

The power of the Principal Principle depends entirely on how much is admissible. If nothing is admissible it is vacuous. If everything is admissible it is inconsistent. (Lewis 1986, p. 92.)

Auxiliary conditions on admissibility must thus tread a careful path between vacuity and inconsistency.

Hawthorne et al. (2017) proposed two such auxiliary conditions. For any contingent atomic proposition $F$,

Condition 1. If $E$ is admissible and $X E$ contains no information that renders $F$ relevant to $A$, then $E F$ is admissible.

Condition 2. If $E$ is admissible and $X E$ contains no information relevant to $F$, then $E(A \leftrightarrow F)$ is admissible.

Hawthorne et al. (2017) show that the Principal Principle-under these auxiliary conditions-implies that $P(F)=0.5$ for the initial credence function $P$. This is a version of the Principle of Indifference.

\subsection{Nisowir}

What motivates the Principal Principle and these auxiliary admissibility conditions? We suggest that the answer is: normal informal standards of what is reasonable ('nisowir' for short). Such standards underlie answers to the above questionnaire, as well as Lewis' own version:

We have some very firm and definite opinions concerning reasonable credence about chance. These opinions seem to me to afford the best grip we have on the concept of chance. (Lewis 1986, p. 84.)

Lewis advocated the Principal Principle largely on the grounds that normal informal standards dictate that one's degrees of belief ought to match chances, insofar as one has evidence of them, in the absence of evidence that defeats this norm-see question (a) above. Lewis also argued that what we call normal informal standards of what is reasonable show that evidence entirely about matters of particular fact at times no later than $t$ is also admissible (Lewis 1986, pp. 85, 92-3). ${ }^{1}$ Similarly, we contend that Condition 1 captures the nisowir that a contingent atomic proposition $F$ would not constitute evidence that defeats the norm, unless there was some further evidence that $F$ is relevant to the proposition $A$ of interest-question (b) exemplifies these standards. Condition 2 captures the nisowir that $A \leftrightarrow F$ would not constitute

\footnotetext{
${ }^{1}$ Nissan-Rozen (2018) recently argued against the admissibility of some historical information that is relevant for explanatory purposes. If this argument is successful, one can restrict such admissibility conditions to propositions that are not explanations.
} 
evidence that defeats the norm, unless there was some further evidence relevant to $F$ itself_-question (c) in the above questionnaire.

Arguably, these principles are doing just what any Bayesian principles should do: they explicate nisowir, in order to develop a formal theory that can provide a guide to life in complex situations, as well as in the simpler situations in which the informal standards themselves suffice.

We take nisowir to be norms of reasonableness that are conformed to widely enough as to be considered normal or standard requirements. Nisowir are informal, but formal explications can be developed to systematise key nisowir. The answers to questions (a)-(c) above, and to questions in Lewis' original questionnaire, are instances of nisowir. The Principal Principle and its auxiliary admissibility conditions are explications of these nisowir. Versions of Bayesianism are neither nisowir nor explications of nisowir-rather, they are complex formal frameworks that are intended to validate and extend key nisowir. Of course, nisowir can also be found in contexts other than discussions of Bayesianism. An example of a nisowir in law is the norm that a defendant in a criminal case should be acquitted if the evidence is wholly insufficient for conviction. In many jurisdictions this nisowir is explicated by rules that permit the judge to acquit the defendant, thereby overriding the subjective judgements of jury members. This is partly to avoid the rare situations in which jurors' subjective judgements fail to conform to this nisowir.

As Lewis observed, auxiliary admissibility conditions need to avoid inconsistency. More generally, if certain nisowir or their explications are found to lead to inconsistency, then one should reject them. ${ }^{2}$ Otherwise, being norms, they ought to be conformed to. The Principal Principle together with Conditions 1 and 2 lead to a result that is perhaps surprising (a version of the Principle of Indifference), but not inconsistent. Hence, credences ought to conform to the Principal Principle with Conditions 1 and 2.

In sum, then, we have certain nisowir about credence, chance and admissibility and the task is to explicate them in such a way as to avoid the extremes of vacuity on the one hand (if auxiliary conditions are too weak, they will fail to explicate our nisowir and will limit the applicability of the Principal Principle) and inconsistency on the other (if auxiliary conditions are too strong, they will generate constraints that cannot jointly be satisfied, in the context of the constraints already imposed by the probability calculus on both chance and credence). Conditions 1 and 2 apparently succeed in this task.

However, this claim has been questioned, as we shall now see.

\footnotetext{
${ }^{2}$ Lewis noted that his Humean metaphysics, when coupled with the above version of the Principal Principle, leads to inconsistency. Lewis' strategy here was to modify the Principal Principle in such a way as to ensure consistency with his Humeanism yet accommodate the nisowir that motivate the Principal Principle (Lewis 1994). Whether or not the new principle successfully validates the nisowir is debatable; if there is no successful explication of the nisowir that is consistent with Humeanism, that would tell against Humeanism.
} 


\section{Pettigrew's dilemma}

Pettigrew has two objections to Conditions 1 and 2, in the form of a dilemma. If Conditions 1 and 2 are supposed to follow from an independent account of admissibility, the first objection applies. Otherwise, if Conditions 1 and 2 constrain any independent account of admissibility, the second objection applies.

We would take the second horn of the dilemma: nisowir constrain formal theory, rather than the other way round. Likewise, Hawthorne et al. (2017) argue that the account of admissibility put forward by Meacham (2010) should be rejected for failing to validate Conditions 1 and 2. Nevertheless, we shall consider each horn in turn.

\subsection{The first horn}

In the first horn of his dilemma, Pettigrew puts forward an account of admissibility, 'Levi-admissibility', that does not validate the nisowir. $E$ is said to be Levi-admissible for a proposition $A$ at time $t$ if and only if for all chance functions $P^{*}$ possible at time $t$ and for which $P^{*}(E)>0, P^{*}(A \mid E)=P^{*}(A)$.

This account of admissibility would be a problem for the argument of Hawthorne et al. (2017), if one could show that Levi-admissibility has as a great a claim to being a standard of what is reasonable as Conditions 1 and 2. Pettigrew does indeed suggest this: 'given the plausible account of admissibility as Levi-admissibility, Conditions 1 and 2 are false and the HLWW argument [i.e., that of Hawthorne et al. (2017)] fails' (Pettigrew 2020, p. 616). However, we shall show that Pettigrew's argument does not succeed.

Pettigrew considers the situation in which $X$ says that the chance of $A$ is 0.5 , where $A$ concerns the toss of a coin in Quito tomorrow, and the question is how strongly to believe $A$ given $X$ and $E$, which is a proposition about tomorrow's weather in Addis Ababa. Pettigrew says:

if I choose to place most of my initial credence on the chance hypotheses on which $E$ is positively relevant to $A$, then my credence in $A$ conditional on $E$ and $[X]$ should not be 0.5 - it should be something greater than 0.5 . (Pettigrew 2020, p. 613.)

Recall that $P$ is an initial credence function-i.e., a belief function in the absence of any evidence-and the Principal Principle constrains certain initial conditional credences. By endorsing Levi's account of admissibility, Pettigrew (2020, p. 613) is suggesting that personal choice with respect to certain other initial credences should render the Principal Principle impotent here, since 'there are certainly possible chance functions - probability functions that, for all she knows, give the objective chances today-that do not render $E$ and $A$ stochastically independent.'

However, this is a fallacious move: it is not reasonable to object to proposed Bayesian constraints on prior belief (here, Conditions 1 and 2) on the grounds that they conflict with uninformed personal choice with respect to other prior beliefs. Consider an analogy. It is a consequence of the norms of Bayesianism that one ought to fully believe the logical truth $A \vee \neg A$. It is clearly not reasonable to object to 
this constraint on the grounds that it conflicts with a personal choice to believe $A$ to degree 0.1 and $\neg A$ to degree 0.3 , say. The fallacy in each case is that of inferring that a constraint on prior beliefs is faulty on the grounds that it conflicts with personal choice with respect to beliefs about other propositions.

Let us consider a possible response to our claim that Pettigrew's move is fallacious: Bayesian Conditionalisation is a principle that determines some degrees of belief on the basis of others, so why shouldn't the Principal Principle do the same? The answer is that there is a crucial disanalogy: Conditionalisation determines later beliefs from a given initial belief function, while the problem here-as Lewis emphasised-is to determine the initial belief function itself. Here, one cannot treat initial beliefs as an input to the problem because they are the output.

In general, then, Pettigrew has $P(A \mid X E)$ depend upon features of the initial credence function, rather than solely on $E$ itself, for any proposition $E$ whose truth is not determined by time $t$. This steers the Principal Principle towards vacuity: it is up to you whether to set your credence to the corresponding chance, i.e., whether to apply the Principal Principle non-vacuously. You can ignore the precept that one should calibrate credences to chances in such a case just by distributing certain other credences appropriately (specifically, credences in the chances being such that $E$ and $A$ are probabilistically dependent). Consider the Quito example. How strongly should you believe that the coin toss in Quito will yield heads tomorrow $(A)$ given that the chance of it doing so is 0.5 (proposition $X$ ) and your other evidence $(E)$ ? Suppose, as a keen windsurfer, your evidence includes the fact that a high tide will occur at your nearest beach at 8.51 am tomorrow, but includes nothing connecting this to the coin toss in Quito, nor anything else relevant to $A$. On Pettigrew's account, you are perfectly reasonable to set $P(A \mid X E)=0.99$, provided you set your credences in the possible chance functions consistently. Thus the Principal Principle has no bite here: you can be deemed to be reasonable whether or not you calibrate your credence in $A$ to the known chance value, 0.5. This does not accord with normal standards of what is reasonable. As our questionnaire illustrates, calibrating credences to known chances in such cases is not merely rationally permissible (as it was anyway, in the absence of the Principal Principle): it is rationally required.

This problem generalises. On Pettigrew's account, a mischief maker can systematically undermine the applicability of the Principal Principle by reliably informing subjects about future tide times. Since Levi-admissibility deems future propositions to be inadmissible evidence, the Principal Principle will fail to fix a value for $P(A \mid X E)$ for any proposition $A$, when $E$ includes a future tide time. Only by rejecting Levi-admissibility and accepting Conditions 1 and 2 can one ensure the applicability of the Principal Principle in the presence of such mischief making.

One might respond that the version of the Principal Principle that Pettigrew invokes does impose substantial constraints, even in the context of a proposition $E$ whose truth is not determined by time $t$. Let proposition $Y_{y}$ assert that the chance of $A$ conditional on $X E$ is $y$. Then,

$$
P(A \mid X E)=\int_{0}^{1} P\left(A \mid X Y_{y} E\right) P\left(Y_{y} \mid X E\right) d y .
$$


In this equation, the version of the Principal Principle endorsed by Pettigrew would motivate setting $P\left(A \mid X Y_{y} E\right)=y$. This appears to be a significant constraint.

However, the values $P\left(Y_{y} \mid X E\right)$ remain unconstrained. For Pettigrew, these values are entirely subjective. Hence, while the above equation can be thought of as a consistency constraint, whether $P(A \mid X E)=x$ depends entirely on the agent in question.

Overall, then, on Pettigrew's account the Principal Principle fails to ensure that the initial conditional credence in $A$ given $X$ and $E$ is calibrated to the chance specified by $X$, when $E$ does not entirely concern matters of fact up to the present. This exposes the Principal Principle to the charge of vacuity. In contrast, $P(A \mid X E)=x$ does indeed hold in key situations when the Principal Principle is taken in conjunction with Lewis' remarks on admissibility and Conditions 1 and 2.

\subsection{The second horn}

Let us turn to the second horn of the dilemma. The structure of this part of Pettigrew's argument is as follows. First, he puts forward some motivation for the following condition:

Condition 2+ If your evidence at $t$ gives no information about the connection between $A$ and $F$, then $A \leftrightarrow F$ is admissible for $A$ at $t$ (for any proposition $F$ ).

Condition $2+$ generalises Condition 2 from an atomic proposition $F$ to an arbitrary proposition $F$. Pettigrew then shows that this generalisation leads to inconsistency in the context of the axioms of probability. He takes this inconsistency to undermine Condition 2 itself.

In response, note that the inconsistency only tells against the generalisation, Condition 2+, and its motivation, not against Condition 2 itself. Here is an analogy. Consider the claim (i) that the union of two sets is a set. One might ask what the motivation for this claim is, and suggest as a generalisation that any union of sets is a set. However, this generalisation leads to inconsistency in the context of the axioms of set theory: in particular it leads to the false claim (ii) that the class of all sets is a set. Clearly, that claim (ii) is false only tells against the generalisation, not against claim (i). It is irrelevant here that one might find claim (ii) to be not much less plausible, prima facie, than claim (i). Claim (ii) leads to inconsistency, while claim (i) is true.

Pettigrew suggests that the onus is on Hawthorne et al. to explain why Condition 2 is true but Condition $2+$ is false. But this is straightforward. Condition $2+$ is false because it leads to inconsistency. Moreover, as question (d) of our questionnaire shows, Condition $2+$ is prima facie dubious. Therefore, Condition $2+$ can hardly be thought of as explicating a nisowir. On the other hand, Conditions 1 and 2 are indeed explications of nisowir, as the questionnaire shows. Furthermore these conditions, apparently, do not lead to inconsistency. Given this, the onus is surely on the detractor to explain why Conditions 1 and 2 are false, if indeed they are false. 
In response, one might suggest on Pettigrew's behalf that their being unproblematic explications of nisowir provides insufficient grounds for accepting Conditions 1 and 2. However, this line of response would ultimately be self-undermining. If a principle cannot be justified on the grounds that it explicates a nisowir, then the Principal Principle itself would lack justification. We saw that for Lewis, the Principal Principle is an attempt to explicate nisowir elicited by a questionnaire similar to that of Section 2. More widely, those who advocate the Principal Principle do so either on the grounds that it directly explicates nisowir, or that there is some argument for the Principal Principle which is itself grounded in other nisowir-e.g., that one should avoid avoidable long-run loss (Williamson 2010, §3.3), or that one should avoid beliefs that are inaccurate (Pettigrew 2012, 2013). Therefore, doubt cast on the importance of nisowir affects the Principal Principle itself, not merely Conditions 1 and 2.

Thus, there is no need for additional general motivation for Conditions 1 and 2 . Any more general motivation for these conditions would ultimately be grounded in nisowir, but the two conditions are already directly motivated by nisowir.

To sum up, neither horn of Pettigrew's dilemma succeeds as an argument against Conditions 1 and 2. The first horn involves an appeal to Levi-admissibility, which fails to validate the important nisowir that underlie Conditions 1 and 2 and which steers the Principal Principle towards vacuity. On the other hand, the considerations underlying the second horn steer the Principal Principle towards inconsistency and fail to undermine Conditions 1 and 2.

\section{Titelbaum and Hart's concerns}

\subsection{Cars, fins and antennae}

The objection of Titelbaum and Hart (2020) appeals to the fact that Condition 2 implies:

$$
P(F \mid A X E)=P(\neg F \mid(\neg A) X E),
$$

where $E$ is admissible and $X E$ contains no information relevant to $F$.

Titlebaum and Hart provide an example involving cars, and whether they have antennae $(A)$ and fins $(F)$ :

HLWW think that armed only with evidence about the proportion of cars with polished antennas $\operatorname{Pr}(A \mid X E)$, and with no evidence about the proportion of cars with polished fins $\operatorname{Pr}(F \mid X E)$, you should assume that

$$
\operatorname{Pr}(F \mid A X E)=\operatorname{Pr}(\neg F \mid(\neg A) X E) .
$$

Yet this strong assumption about the proportions of polished cars hardly seems justified by the state of ignorance in which you find yourself.

Equation (1) captures HLWW's core intuition (Titelbaum and Hart 2020, p. 623). 
The argument is thus that Hawthorne et al. (2017) assume (1), which says something implausible about empirical proportions. ${ }^{3}$ However, this argument is problematic for the following reason.

Condition 2 is a statement about admissibility. It implies (1), which is a statement about rational degrees of belief. ${ }^{4}$ However, (1) does not imply (2), which is interpreted as a statement about empirical proportions. Indeed 'this strong assumption about the proportions of polished cars hardly seems justified' (Titelbaum and Hart 2020, p. 623) because it is not justified. But that hardly matters, because Hawthorne et al. (2017) do not claim that (2) holds. To take (1) to imply (2) is an instance of what Jaynes (2003) calls the 'Mind Projection Fallacy': all (1) says is that two credences coincide. (Analogously, that one believes that an experiment will have a positive outcome to degree $\frac{1}{2}$ does not imply that the proportion of experiments yielding a positive outcome is $\frac{1}{2}$.)

Now, Titelbaum and Hart might object that, in the context of the Principal Principle, it is possible to interpret at least some credences as saying something about chances or proportions. ${ }^{5}$ For example, if $P(A \mid X E)=y$ then one can infer that $P^{*}(A)=y$, where $P^{*}$ denotes the chance function, because if $P(A \mid X E)=y$, the Principal Principle holds, and $X$ says that $P^{*}(A)=x$, then $x=y$.

However, this line of reasoning extends neither to (1) nor to Condition 2. Consider (1): $P(F \mid A X E)$ says nothing about $P^{*}(F \mid A)$ because $X E$ says nothing about the chance of $F$; similarly for $P(\neg F \mid(\neg A) X E)$. To take these statements as saying anything about chances or proportions remains problematic.

In sum, Titelbaum and Hart's claim is that Hawthorne et al. (2017) assume (2), which says something implausible about proportions. As we have seen, however, nothing that Hawthorne et al. (2017) say implies anything about (2).

One might respond that while (1) does not say anything about proportions, it nevertheless represents a very strong position. But in the Bayesian framework, every assignment of degrees of belief represents an equally substantive position, insofar

\footnotetext{
${ }^{3}$ In their discussion, Titelbaum and Hart "use the $P r$ function to stand for numerical proportions, chances, and rational credences." This is because, they claim, "In the example we've constructed such values are uncontroversially interchangeable" (Titelbaum and Hart 2020, Footnote 3). We disagree. As we explain below, these quantities are not interchangeable, especially in the context of debates about the Principal Principle. Given this conflation of proportion, chance and credence, one can only guess at the intended meaning of Titelbaum and Hart's argument. It seems plausible though that $P r$ is being used to refer to empirical proportions (i.e., actual frequencies) in this passage.

${ }^{4}$ Note that (1) is not an assumption-it is a consequence of an assumption. Propositional attitudes (assumption, in this case) need not be closed under logical consequence. Just as you might not believe all the consequences of your beliefs, you might not assume all the logical consequences of your assumptions. Worse, while perhaps you ought to believe the consequences of your beliefs (or your assumptions), it is clearly not the case that you ought to assume the consequences of your assumptions. Hence, (1) cannot be interpreted as an assumption. In addition, if a consequence of an assumption is counterintuitive or implausible, that does not necessarily imply that the original assumption should be rejected. That depends on how intuitive or plausible the original assumption is itself, and on its other consequences. So, if (1) were implausible that would not necessarily warrant rejecting Condition 2. Condition 2 may be more plausible than (1) is implausible, in virtue of the nisowir that Condition 2 explicates.

${ }^{5}$ Indeed Lewis (1986, p. 88) suggested that chances might be even be analysable in terms of reasonable initial credences.
} 
as it is a commitment to betting or decision-making behaviour. $P(F \mid A X E)=$ $P(\neg F \mid(\neg A) X E)$ is no more of a commitment than $P(F \mid A X E)=z$, for any other value $z$. The only way to avoid making such commitments is to move to another framework, such as that of imprecise probability, where the connection between strength of belief and decision making is less perspicuous.

Titelbaum and Hart conclude by claiming that whether a proposition is admissible depends at least partly on its initial probability. They do not spell out an account of admissibility, but what they do say reintroduces the same sort of worries as those that apply to Levi-admissibility: the danger is that one can play around with the initial probabilities to strip the Principal Principle of (some of its) bite.

Thus, Titelbaum and Hart's main reason for rejecting Condition 2 hinges on failing to properly differentiate between chances and credences or an application of the Mind Projection Fallacy and so is unconvincing. It remains to be seen as to whether there are genuine grounds for thinking Condition 2 is false. ${ }^{6}$

\subsection{At the races}

Hart and Titelbaum (2015) put forward an example, At the Races, which might suggest that rather than implying it, Condition 2 is incompatible with the Principle of Indifference. $^{7}$

Suppose your credence that a toss with a fair coin comes up heads is $\frac{1}{2}$, i.e., $P(A \mid X E)=\frac{1}{2}$ where $X$ says that $P^{*}(A)=\frac{1}{2}$ and $E$ is admissible. The proposition $F$ concerns the outcome of a horse race with six participating horses and says that Speedy wins the race. You know nothing about horses nor horse races, and conform to the Principle of Indifference by setting $P(F \mid X E)=\frac{1}{6}$. You then learn that Speedy won, if and only if the toss landed heads, i.e., $A \leftrightarrow F$.

Hart and Titelbaum prove that if $P(F \mid X E)=\frac{1}{6}$, then $P(A \mid X E(A \leftrightarrow F))=$ $\frac{1}{6}$. So it seems that Condition 2 is incompatible with the Principle of Indifference, because Condition 2 would imply $P(A \mid X E(A \leftrightarrow F))=\frac{1}{2}$.

However, this conflict between Condition 2 and the Principle of Indifference is only illusory. Condition 2 requires that $F$ is contingent and atomic, $E$ is admissible, and $X E$ contains no information that renders $F$ relevant to $A$. Here, however, the proposition $F$ is true just when one of six mutually exclusive and exhaustive outcomes takes place. This information can be taken into account in one of two ways. Either $F$ states that Speedy wins and none of the other horses wins, or $F$ states merely

\footnotetext{
${ }^{6}$ Titelbaum and Hart do correctly point to a technical deficiency of Hawthorne et al. (2017), who say: 'If one gives higher prior probability to $\neg A F$ than one does to $A \neg F$, then $A \leftrightarrow F$ does apparently favour $A$ over $\neg A$. Similarly, if $\neg A F$ has lower prior probability than $A \neg F$ then $A \leftrightarrow F$ apparently favours $\neg A$ over $A$.'

This should have read: 'If one gives higher prior probability to $A F$ than one does to $\neg A \neg F$, then $A \leftrightarrow F$ does apparently favour $A$ over $\neg A$. Similarly, if $A F$ has lower prior probability than $\neg A \neg F$ then $A \leftrightarrow F$ apparently favours $\neg A$ over $A$.'

Indeed, $P(A \mid A \leftrightarrow F)>P(\neg A \mid A \leftrightarrow F)$ iff $P(A F)>P(\neg A \neg F)$.

${ }^{7}$ Strictly speaking, Hart and Titelbaum (2015) object to an argument of Roger White (2010) rather than Condition 2. However, the assumption they attack, i.e., Equation (5) on p.175, is similar to Condition 2.
} 
that Speedy wins but there is additional background information $E$ which says that precisely one horse can win. In the former case $F$ is logically complex, while in the latter case $E$ contains information relevant to $F$. Either way, the antecedent of Condition 2 is false, hence Condition 2 fails to constrain $P(A \mid X E(A \leftrightarrow F))$. Hence, $A t$ the Races is not relevant to Condition 2, after all.

\section{Conclusion}

Following Lewis, we have suggested that the Principal Principle and its auxiliary admissibility conditions need to explicate normal informal standards of what is reasonable. These principles also need to steer a course between vacuity and inconsistency. Given these desiderata, Conditions 1 and 2 are hard to resist: they do indeed explicate nisowir and they are strong conditions, yet apparently not so strong as to lead to inconsistency. It is for these reasons that Conditions 1 and 2 hold, and for these reasons that the Principal Principle implies the Principle of Indifference.

We have seen that, given the motivation in terms of nisowir, the second horn of Pettigrew's dilemma applies, rather than the first. On this second horn, Pettigrew demands some motivation for Condition 2 which does not appeal to general considerations that lead to inconsistency. But this is straightforward: Condition 2 explicates a nisowir. Titelbaum and Hart worry that Condition 2 makes unjustified assumptions about proportions. On the contrary, it assumes nothing about proportions, and what it says about credences is justified by nisowir. Again, Condition 2 is not so easy to resist.

We note a further objection, due to Gyenis and Wroński (2017). They suggest that it is not the Principal Principle that implies the Principle of Indifference, but rather a set of probabilistic independence constraints, which need not even mention the Principal Principle. In response, we would simply observe that it is the Principal Principle together with auxiliary Conditions 1 and 2 that guarantee that these independence constraints routinely hold. Since nisowir ground the Principal Principle and auxiliary Conditions 1 and 2, the conclusion of the argument-the Principle of Indifference-is well motivated.

One might employ a sort of reductio ad absurdum to argue that the implausibility of the conclusion of the argument undermines the nisowir that motivate the Principal Principle and its auxiliary conditions. ${ }^{8}$ Indeed, the Principle of Indifference is notoriously problematic, both on continuous domains, where there are multiple ways of being indifferent, and on finite domains, where different domains lead to different assignments of credences. In response, however, it is sufficient to point out that these concerns with the Principle of Indifference do not affect the use that is made of it here. First, the partition of interest, $\{F, \neg F\}$ is finite, not continuous. Second, the domain is fixed: the agent has a fixed language at the time in question and this language determines whether $F$ is an atomic proposition, so there is no question of

\footnotetext{
${ }^{8}$ Similarly, Dylan Dodd (2012) argues that White's premisses imply a version of Bertrand's paradox and therefore rejects White's argument.
} 
the agent having different credences in the same proposition at the same time. That different agents with different languages might have different credences in the same proposition at the same time is hardly cause for concern in the Bayesian framework.

While reductio ad absurdum cannot successfully undermine the argument from the Principal Principle to the Principle of Indifference, one might nevertheless challenge it by observing that one person's modus ponens is another's modus tollens. Thus if PP denotes the Principal Principle and its auxiliary admissibility conditions, and PoI the version of the Principle of Indifference that they imply, an ardent subjectivist Bayesian may reconstrue $\mathrm{PP} \Rightarrow \mathrm{PoI}$ as the contrapositive $\neg \mathrm{PoI} \Rightarrow \neg \mathrm{PP}$ and take her subjectivism as reason enough to reject PP, and Conditions 1 and 2 in particular. The arguments of this paper provide grounds for resisting such a reconstrual. We have argued that PP explicates nisowir - the nisowir exemplified by the questionnaire of Section 2. On the other hand, $\neg$ PoI does not explicate a nisowir: the claim that one is not rationally required to believe an atomic proposition to the same degree as its negation, in the absense of any evidence pertinent to that proposition, is hardly a normal informal standard of what is reasonable. That PP aligns more closely to normal informal standards than $\neg \mathrm{PoI}$ favours the modus ponens involving $\mathrm{PP} \Rightarrow \mathrm{PoI}$ over the modus tollens involving $\neg \mathrm{PoI} \Rightarrow \neg \mathrm{PP}$.

That one person's modus ponens is another's modus tollens is the bane of philosophy. It can be wheeled out against almost any philosophical argument, and is perhaps more pernicious than epistemological scepticism, which has few if any genuine adherents. However, an appeal to normal informal standards of what is reasonable can sometimes come to the rescue, where an appeal to mere intuition cannot. An argument of the form ' $A \Rightarrow B$, intuitively $A$, therefore $B$ ', can be resisted by someone who simply denies $B$, by disavowing the intuition about $A$. This is not so in the case of an argument of the form ' $A \Rightarrow B, A$ is (an explication of) a nisowir, therefore $B$ '. Either $A$ captures a nisowir or it doesn't: this is not a matter of subjective intuition. ${ }^{9}$ Thus nisowir may have a wider role to play in reinforcing philosophical argumentation.

Acknowledgements We are grateful to the anonymous referees for very helpful comments.

Funding Open Access funding enabled and organized by Projekt DEAL. Jon Williamson's research was supported by Leverhulme Trust grant RPG-2019-059. Jürgen Landes gratefully acknowledges funding from the European Research Council (grant 639276) and from the Deutsche Forschungsgemeinschaft (DFG, German Research Foundation) 405961989 and 432308570 . Christian Wallmann gratefully acknowledges funding from the Austrian Federal Ministry for Digital and Economic Affairs; the Austrian National Foundation for Research, Technology and Development.

\section{Declarations}

Conflict of Interests The author(s) declare that he/she/it/they has/have no conflict of interest.

\footnotetext{
${ }^{9}$ As we have seen, questionnaires can be used to elicit nisowir. One might use experimental philosophy to determine more rigorously whether a particular nisowir holds.
} 
Open Access This article is licensed under a Creative Commons Attribution 4.0 International License, which permits use, sharing, adaptation, distribution and reproduction in any medium or format, as long as you give appropriate credit to the original author(s) and the source, provide a link to the Creative Commons licence, and indicate if changes were made. The images or other third party material in this article are included in the article's Creative Commons licence, unless indicated otherwise in a credit line to the material. If material is not included in the article's Creative Commons licence and your intended use is not permitted by statutory regulation or exceeds the permitted use, you will need to obtain permission directly from the copyright holder. To view a copy of this licence, visit http://creativecommonshorg/licenses/by/4. $0 \%$.

\section{References}

Black, R. (1998). Chance, credence, and the principal principle. British Journal for the Philosophy of Science, 49(3), 371-385.

Decock, L., Douven, I., Sznajder, M. (2016). A geometric principle of indifference. Journal of Applied Logic, 19, 54-70.

Dodd, D. (2012). Roger White's argument against imprecise credences. British Journal for the Philosophy of Science, 64(1), 69-77.

Easwaran, K., \& Fitelson, B. (2012). An "Evidentialist" worry about joyce's argument for probabilism. Dialectica, 66(3), 425-433.

Gyenis, B., \& Wroński, L. (2017). Is it the principal principle that implies the principle of indifference?. In Hofer-Szabó, G., \& Wroński, L. (Eds.) Making it Formally Explicit (pp. 35-41). Cham: Springer International Publishing.

Hall, N. (1994). Correcting the guide to objective chance. Mind, 103(412), 505-518.

Halpern, J.Y., \& Koller, D. (2004). Representation dependence in probabilistic inference. Journal of Artificial Intelligence Research, 21, 319-356.

Hart, C., \& Titelbaum, M.G. (2015). Intuitive dilation?. Thought: A Journal of Philosophy, 4(4), $252-262$.

Hawthorne, J., Landes, J., Wallmann, C., Williamson, J. (2017). The Principal Principle implies the Principle of Indifference. British Journal for the Philosophy of Science, 68, 123-131.

Jaynes, E.T. (2003). Probability theory: the logic of science. Cambridge: Cambridge University Press.

Lewis, D.K. (1986). A Subjectivist's guide to objective chance. In Philosophical papers, (Vol. 2 pp. 83132). Oxford: Oxford University Press. With postscripts.

Lewis, D.K. (1994). Humean supervenience debugged. Mind, 412, 471-490.

Marcoci, A. (2020). Monty hall saves dr. Evil: on elga's restricted principle of indifference. Erkenntnis, $85(1), 65-76$.

Meacham, C.J.G. (2010). Two mistakes regarding the principal principle. British Journal for the Philosophy of Science, 61(2), 407-431.

Nissan-Rozen, I. (2018). On the inadmissibility of some historical information. Philosophy and Phenomenological Research, 97(2), 479-493.

Norton, J.D. (2019). A demonstration of the incompleteness of calculi of inductive inference. British Journal for the Philosophy of Science, 70, 1119-1144.

Novack, G. (2010). A defense of the principle of indifference. Journal of Philosophical Logic, 39(6), 655-678.

Paris, J.B. (2006). The Uncertain Reasoner's Companion: A Mathematical Perspective, volume 39 of Cambridge Tracts in Theoretical Computer Science, 2nd edn. Cambridge: Cambridge University Press.

Paris, J.B. (2014). What you see is what you get. Entropy, 16(11), 6186-6194.

Pettigrew, R. (2012). Accuracy, chance, and the principal principle. The Philosophical Review, 121(2), 241-275.

Pettigrew, R. (2013). A new epistemic utility argument for the principal principle. Episteme, 10(1), 19-35.

Pettigrew, R. (2016). Accuracy, risk, and the principle of indifference. Philosophy and Phenomenological Research, 92(1), 35-59.

Pettigrew, R. (2020). The Principal Principle does not imply the Principle of Indifference. British Journal for the Philosophy of Science, 71(2), 605-619.

Rinard, S. (2014). The principle of indifference and imprecise probability. Thought, 3(2), 110-114. 
Seidenfeld, T. (1987). Entropy and uncertainty. In MacNeill, I.B., Umphrey, G.J., Safiul Haq, M., Harper, W.L., Provost, S.B. (Eds.) Advances in the statistical sciences: foundations of statistical inference, (Vol. 35 pp. 259-287): Springer.

Smithson, R. (2017). The principle of indifference and inductive scepticism. British Journal for the Philosophy of Science, 68(1), 253.

Titelbaum, M.G., \& Hart, C. (2020). The principal principle does not imply the principle of indifference, because conditioning on biconditionals is counterintuitive. British Journal for the Philosophy of Science, 71(2), 621-632.

Vranas, P.B.M. (2004). Have your cake and eat it too: the old principal principle reconciled with the new. Philosophy and Phenomenological Research, 69(2), 368-382.

Wallmann, C., \& Hawthorne, J. (2018). Admissibility troubles for Bayesian direct inference principles. Erkenntnis, pp. 1-37.

Wallmann, C., \& Williamson, J. (2020). The Principal Principle and subjective Bayesianism. European Journal for the Philosophy of Science, 10(1), 3.

White, R. (2010). Evidential symmetry and mushy credence. Oxford studies in epistemology, 3, 161-86.

Williamson, J. (2010). In defence of objective Bayesianism. Oxford: Oxford University Press.

Williamson, J. (2018). Justifying the principle of indifference. European Journal for Philosophy of Science, pp. 559-586.

Publisher's note Springer Nature remains neutral with regard to jurisdictional claims in published maps and institutional affiliations. 